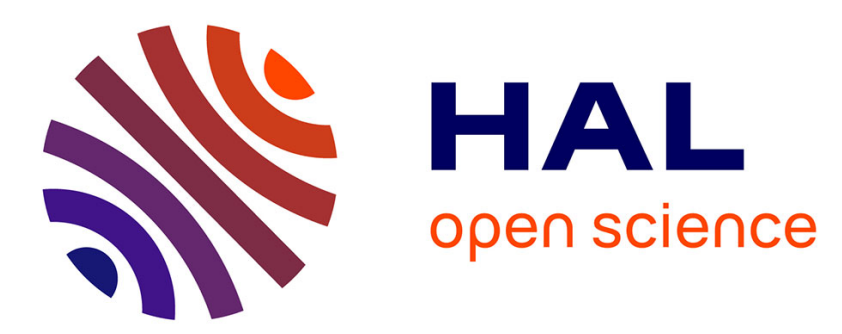

\title{
Spin-transfer Torque and Topological Changes of Magnetic Textures
}

Alberto Verga

\section{To cite this version:}

Alberto Verga. Spin-transfer Torque and Topological Changes of Magnetic Textures. Discontinuity, Nonlinearity, and Complexity, 2016, 5 (1), pp.19-24. 10.5890/DNC.2016.03.003 . hal-01500449

\section{HAL Id: hal-01500449 \\ https://hal-amu.archives-ouvertes.fr/hal-01500449}

Submitted on 3 Apr 2017

HAL is a multi-disciplinary open access archive for the deposit and dissemination of scientific research documents, whether they are published or not. The documents may come from teaching and research institutions in France or abroad, or from public or private research centers.
L'archive ouverte pluridisciplinaire HAL, est destinée au dépôt et à la diffusion de documents scientifiques de niveau recherche, publiés ou non, émanant des établissements d'enseignement et de recherche français ou étrangers, des laboratoires publics ou privés. 


\title{
Spin-transfer torque and topological changes of magnetic textures
}

\author{
Alberto Verga \\ Aix-Marseille Université, IM2NP, Campus St Jérôme, service 142, \\ 13387 Marseille, France
}

\begin{abstract}
The electric manipulation of magnetic textures in nanostructures, important for applications in spintronics, can be realized through the spin-transfer torque mechanism: a spin-polarized current can modify the magnetization of skyrmions and magnetic vortices, and eventually change the topology of the magnetization. The spin-transfer torque and the intrinsic space and time scales of the topological changes are essentially quantum mechanical. We model the interaction between itinerant and fixed spins with a simple tight-binding hamiltonian in a square lattice. The dynamics is described by the Schrödinger equation for the electrons and the Landau-Lifshitz equation for the evolution of the magnetic texture. We investigate the phenomenology of the topological change of a Belavin-Polyakov skyrmion under the action of a spin-polarized current and show that adding an exchange dissipation term, regularizes the transition towards a ferromagnetic state.
\end{abstract}

Keywords: Magnetism, magnetic vortices, Landau-Lifshitz equation, spin-transfer torque

\section{INTRODUCTION}

The equilibrium magnetization field in ferromagnetic nanodots and in helical metals, often possesses a nontrivial topology. Magnetic vortices in permalloy [Van Waeyenberge et al. (2006)] and skyrmion lattices in transition metal compounds [Muhlbauer et al. (2009)] were experimentally observed. These configurations are interesting because a change between states having different topologies (switching of the vortex core, motions and annihilation of skyrmions), can be used as basic states in nonvolatile memories and spintronic devices [Romming et al. (2013)]. The basic physical mechanism that can trigger these topological changes, without magnetic fields, is the spin-transfer torque [Ralph and Stiles (2008)]. It describes the interaction between itinerant spins $s$, produced by a spin-polarized current $\boldsymbol{j}_{s}$, and the magnetic moments (fixed spins $\boldsymbol{S}$ ) of the magnetic material, when the underlying magnetization is non-uniform. Within the framework of micromagnetism, to account for the spin-transfer torque one has to extend the usual Landau-Lifshitz equation [Landau and Lifshitz (1935)] with terms proportional to the current and the magnetization gradients $\boldsymbol{j}_{s} \cdot \nabla \boldsymbol{S}$ [Zhang and Li (2004)]. However, this approach neglects strong non-adiabatic effects, such as the generation of current inhomogeneities due the scattering of electrons on the magnetization gradients. In order to investigate these effects, we recently proposed a self-consistent model, where electrons obey to quantum dynamics [Elías and Verga (2014)]. Here we develop further this model, and investigate the influence of dissipation.

In this paper we are interested in the skyrmion-ferromagnetic transition induced by a polarized current. The basic magnetic texture is taken to be a stabilized lattice version of the Belavin-Polyakov [Belavin and Polyakov (1975)] skyrmion in a periodic system. After a presentation of the basic equations coupling the Schrödinger equation for the itinerant spins (electrons) to the Landau-Lifshitz equation for the ferromagnetic spins (fixed classical magnetic moments), defined on a square lattice, we present a qualitative model to show the basic mechanism of the transition. The transition from the skyrmion state to the ferromagnetic state implies a change in topology, and thus a violation of the topological charge conservation. This is possible because of the large fluctuations of the electron spin field (it acts a stochastic perturbation to the magnetization field) and because of the discreteness of the system (the lattice constant introduces a microscopic length cutoff). We performed a series of numerical computations to study the phenomenology of the transition and to identify the mechanism of the topological change.

\section{MODEL}

The motion of an electron in a lattice of step $a$ and size $L^{2}$ is given by the Heisenberg equation for the two components (for the spin up and down) annihilation operator $c_{i}$ at site $i$,

$$
\mathrm{i}_{i}(t)=H_{e}\left(t, S_{i}\right) c_{i}(t)
$$

where $H_{e}$ is the time dependent hamiltonian,

$$
\begin{aligned}
H_{e}=-\epsilon \sum_{\langle i, j\rangle} \mathrm{e}^{\mathrm{i} \phi_{i, j}(t)} c_{i}^{\dagger} c_{j}-J_{s} \sum_{i} \boldsymbol{S}_{i} \cdot\left(c_{i}^{\dagger} \boldsymbol{\sigma} c_{i}\right) \\
-\boldsymbol{B}_{p} \cdot \sum_{i} c_{i}^{\dagger} \boldsymbol{\sigma} c_{i}
\end{aligned}
$$

where the fixed $\boldsymbol{S}_{i}$ and itinerant spins $c_{i}^{\dagger} \boldsymbol{\sigma} c_{i}$ ( $\boldsymbol{\sigma}$ is the vector of Pauli matrices) are coupled by the exchange constant $J_{s} ; \epsilon$ is the energy to jump from site $i=$ $\left(x_{i}, y_{i}\right) / a=\boldsymbol{x}_{i} / a$ to its neighbor $j$. The system is subject to a constant electric field $E$ in the $x$-direction, responsible of the phase factor appearing in the kinetic energy term, 
$z / \lambda$

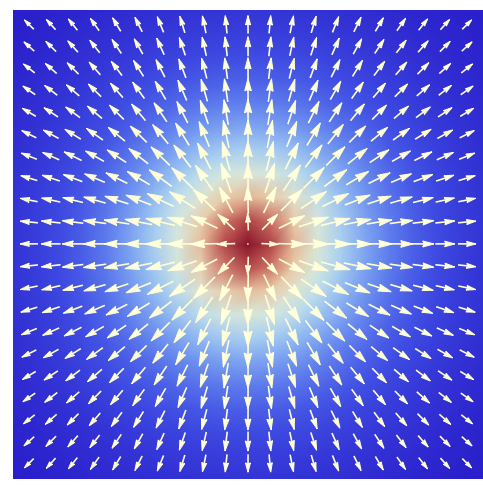

$(1+i) z / \lambda$

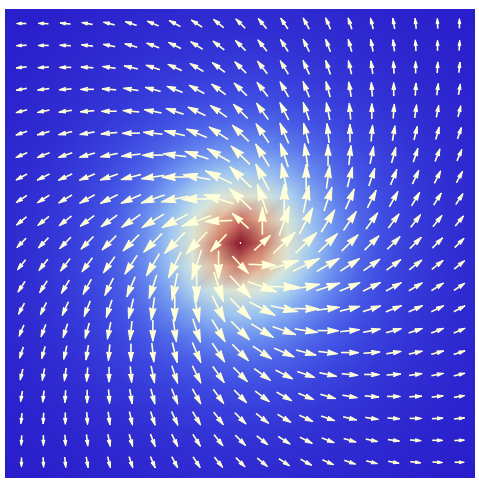

$(1-i) z / \lambda$

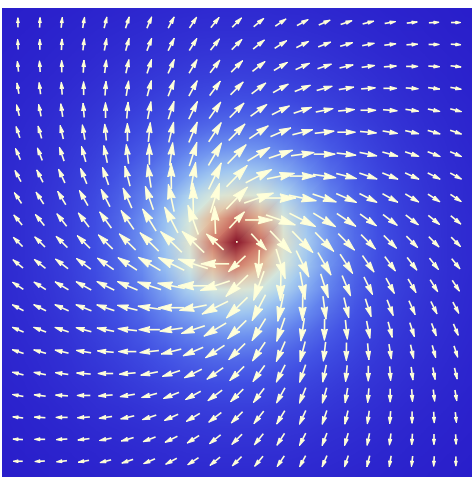

Figure 1. Skyrmions of topological charge $Q=1$ and size $\lambda$ defined by the stereographic projection on the complex plane $w=w(z)$, for (left) $w=z / \lambda$, (center) $w=(1+\mathrm{i}) z / \lambda$, and (right) $w=(1-\mathrm{i}) z / \lambda$. Arrows give the magnetization in the plane, and color, from blue to red, gives its $z$-component: at the center $\boldsymbol{S}=(0,0,1)$. A constant $z$-polarized current induces the precession of the skyrmion magnetization and tends to reduce it core size.

$$
\phi_{i, j}(t)=(i-j) \cdot \hat{x} \frac{e a E t}{\hbar}
$$

(with $-e$ the electron charge). The last term in (2) contains the current polarization effective magnetic field $\boldsymbol{B}_{p}$ in energy units.

The magnetic texture follows the dynamics given by the Landau-Lifshitz equation,

$$
\frac{\partial}{\partial t} \boldsymbol{S}_{i}=\boldsymbol{S}_{i} \times\left(\boldsymbol{f}_{i}-\alpha \boldsymbol{S}_{i} \times \boldsymbol{f}_{i}+J_{s} \boldsymbol{s}_{i}\right)-\beta \nabla^{2} \boldsymbol{f}_{i},
$$

where the effective field,

$$
\boldsymbol{f}_{i}=-\frac{\delta H_{S}}{\delta \boldsymbol{S}_{i}},
$$

is derived from the coarse-grained free energy,

$$
H_{S}=\frac{J}{2} \sum_{i}\left(\nabla \boldsymbol{S}_{i}\right)^{2}+\frac{K}{2} \sum_{i} S_{z i}^{2}-\frac{D}{2} \sum_{i} \boldsymbol{S}_{i} \cdot\left(\nabla \times \boldsymbol{S}_{i}\right)
$$

$J$ is the Heisenberg exchange constant, $K$ is the anisotropy (easy-plane if positive, and easy-axis if negative), and $D$ is the Dzyaloshinski-Moriya spin-orbit coupling energy. The term in $\beta$ represents a dissipation of the magnetization related to the exchange energy (proportional to the gradients $\nabla \boldsymbol{S}_{i}$ ) [Baryakhtar et al. (1997)]. This equation is coupled to the equation for the electrons through the torque in $J_{s}$, where the electron spin is computed by the formula $\boldsymbol{s}_{i}(t)=\left\langle c_{i}^{\dagger}(t) \boldsymbol{\sigma} c_{i}(t)\right\rangle$ where the braket is for the quantum mean value. We use periodic boundary conditions in order to have a well defined topology. Note that $\nabla$ is a difference operator acting on the lattice sites. In practice, the difference operators are computed in Fourier space and transformed to the lattice space. In units such that $\epsilon=a=\hbar=e=1$, typical parameters are: $J_{s}=1$, $J=0.4, K=D=0$ (for the Belavin-Polyakov skyrmion), $B_{p}=n_{e}=0.1, L=128$.

Without the dissipation term, the system (1-5) conserves the magnetization modulus $|\boldsymbol{S}|=1$, and the topological charge $Q=Q(t)$,

$$
\begin{array}{r}
Q=\int \frac{d \boldsymbol{x}}{4 \pi} q(\boldsymbol{x}, t), \quad Q_{+}=\int \frac{d \boldsymbol{x}}{4 \pi}|q(\boldsymbol{x}, t)|, \\
q=\boldsymbol{S} \cdot \partial_{x} \boldsymbol{S} \times \partial_{y} \boldsymbol{S},
\end{array}
$$

where the integration is over the lattice; $Q_{+}(t)$ is a rough measure of the number of vortices.

\section{SKYRMION}

To study the skyrmion-ferromagnetic transition it is convenient to work in the continuous limit, and to transform the magnetization field (which has only two independent components) by the stereographic projection:

$$
S_{x}=\frac{w+\bar{w}}{1+|w|^{2}}, S_{y}=\frac{1}{\mathrm{i}} \frac{w-\bar{w}}{1+|w|^{2}}, S_{z}=\frac{1-|w|^{2}}{1+|w|^{2}},
$$

with

$$
w=\frac{S_{x}+\mathrm{i} S_{y}}{1+S_{z}} .
$$

When $S_{z}=1, w$ goes to zero, and in the opposite pole, $S_{z}=-1, w$ goes to infinity. This projection maps the vector field over the unit sphere $\boldsymbol{S}=\boldsymbol{S}(\boldsymbol{x}, t)$ to the field over the complex plane $w=w(z, \bar{z}, t)$ (where $z=x+\mathrm{i} y$ ). The Landau-Lifshitz equation, in absence of dissipation, becomes,

$$
\begin{aligned}
\mathrm{i} \partial_{t} w=-J \partial \bar{\partial} w+\frac{2 J \bar{w}}{1+|w|^{2}} \partial w \bar{\partial} w & \\
& -\frac{1}{2} s_{+}+s_{z} w+\frac{1}{2} s_{-} w^{2},
\end{aligned}
$$

(only the exchange field is considered here) where we defined the complex derivative $\partial=\partial / \partial x-\mathrm{i} \partial / \partial y$ an its complex conjugate $\bar{\partial}$. The second line in (9) corresponds to the spin-transfer torque term, where $s_{ \pm}=s_{x} \pm \mathrm{i} s_{y}$. Equilibrium solutions of (9) are arbitrary analytic functions $w=w(z)$ and $s=0$. The simplest one is the simple zero, $w=z / \lambda$, the Belavin-Polyakov skyrmion of charge $Q=1$, centered at the origin and of characteristic size $\lambda \in \mathbb{R}$ (spin up at the origin and spin down at infinity).

In order to investigate how the spin torque perturbs the skyrmion state $w=z / \lambda$, we focus on two simple limiting cases: first, small deviations from the skyrmion state by a uniform polarized current $s=\left(0,0, s_{z}\right)$; and second, a small circular region around the skyrmion core relevant to track the transition towards the ferromagnetic state $|w| \rightarrow \infty$. 

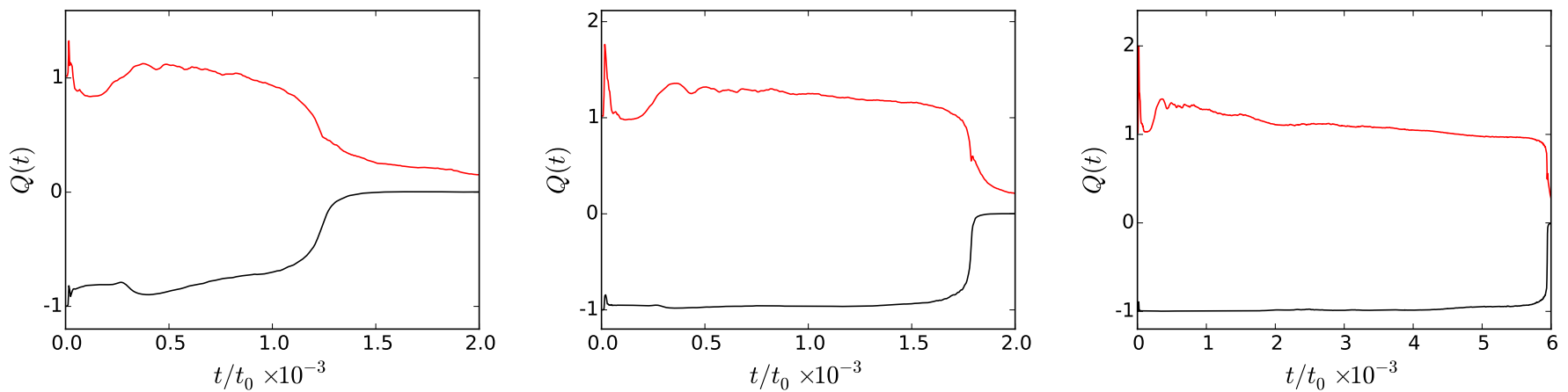

Figure 2. Topological charge $Q$ as a function of time (in adimensional units) for different values of the exchange dissipation parameter: (left) $\beta=0.1$, (center) $\beta=0.01$, and (right) $\beta=0.001$. The red line is for $Q_{+}$. The initial skyrmion charge is $Q=-1$, and the current is polarized in the $-z$ direction. The change from $Q=-1$ to $Q=0$ corresponds to a transition from the skyrmion to the ferromagnetic sate. Decreasing the dissipation strength results in an increase of the time necessary to reach the transition: $t=1236,1748$ and 5936 , for $\beta=0.1,0.01$ and 0.001 , respectively. The electric field is $E=10^{-3}$.
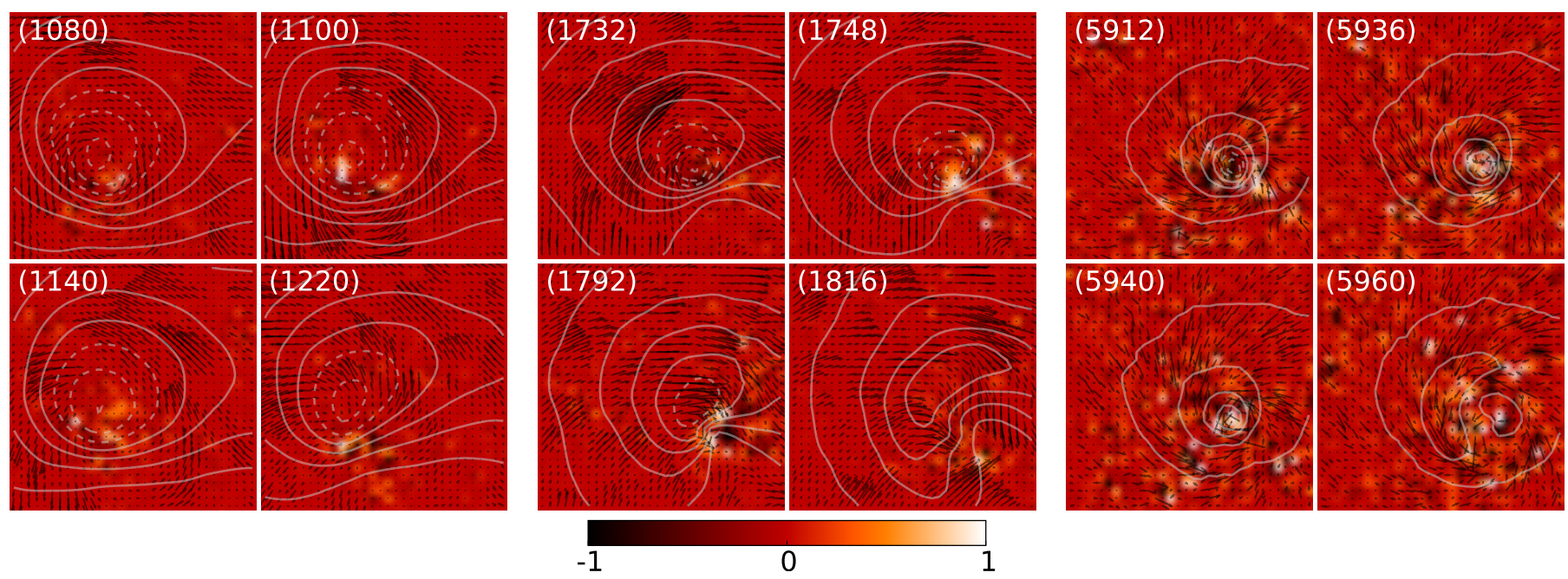

Figure 3. Phenomenology of the topological transition. Contours of $S_{z}$ (fixed spins), arrows of ( $\left.s_{x}, s_{y}\right)$ (itinerant spins), and color density of the topological $b$-field. The dissipation is (left) $\beta=0.1$, (center) $\beta=0.01$, and (right) $\beta=0.001$. The transition towards the ferromagnetic state is correlated with the appearance of intense $b$-field structures possessing a topological charge opposite to one of the skyrmion texture.

In the first case, we linearize (9) around the skyrmion state, $w=z / \lambda+f(z, \bar{z}, t)$ :

$$
\mathrm{i} \partial_{t} f=-J \partial \bar{\partial} f+\frac{2 J \bar{z}}{\lambda^{2}+|z|^{2}} \bar{\partial} f+\frac{s_{z}}{\lambda} z
$$

the spin torque appears as a source term in this approximation. An interesting particular solution is readily found:

$$
f=f_{0}(z, t)=-\mathrm{i} \frac{s_{z} t}{\lambda} z
$$

The pure imaginary factor has the effect of changing the orientation of the magnetization field around the center of the skyrmion, passing successively in time from left to right chirality (as shown in Fig. 1). A sequence observed in the numerical simulations, in addition to the fact that the effective size of the core reduces: $\lambda \rightarrow \lambda / \sqrt{1+\left(s_{z} t\right)^{2}}$ (even if, for long times, the perturbation analysis ceases its validity).

We turn now to the second case, for which we assume that the collapse of the skyrmion core is radially symmetric $w=w(r, t)$, with $r$ the polar radius. We are interested in the asymptotic limit of very large $|w|$, and propose a self-similar form [Zakharov (1972)]:

$$
w(r, t)=\frac{1}{\left(t_{*}-t\right)^{\alpha}} f\left(\frac{r}{\left(t_{*}-t\right)^{\beta}}\right)
$$

to describe the approach of $w$ to infinity (when $t \rightarrow t_{*}, t_{*}$ is the collapse time). Equation (9) becomes,

$$
\mathrm{i} \partial_{t} w(r, t)=-\frac{J}{r} \partial_{r}\left(r \partial_{r} w\right)+\frac{2 J}{w}\left(\partial_{r} w\right)^{2}+\frac{1}{2} s_{-} w^{2}
$$

Inserting ansatz (11) into (12) one obtains, two conditions that determine the unknown exponents:

$$
\alpha=1, \quad \beta=1 / 2 \text {. }
$$

It is worth noting that the exchange interaction, which is scale invariant, do not permit to select the $\alpha$ exponent; its value is determined by the coupling term with the spin polarized current. A crude estimation of the finite time singularity is $t_{*} \sim \lambda / s_{0} a$, where $s_{0} \sim n_{e} B_{p}$ is the typical itinerant spin strength per site. 


\section{RESULTS}

We investigate now, the role of dissipation in the transition towards the ferromagnetic state, using the numerical computation of equations (1) and (3). The first effect of the dissipation is to break the invariance of the topological charge. We observe in particular that the exchange dissipation term favorises the transition from the skyrmion state to the ferromagnetic state. Indeed, we plot in Fig. 2 the evolution of the topological charge as a function of the dissipation strength. In addition to the increasing stability of the skyrmion state, it is worth noting that the characteristic transition time sharply increases with dissipation. This is, as expected, a regularization effect. In the limit of vanishing dissipation the transition produces, ultimately, by the change of a single spin.

A quantitative explanation of the mechanism behind the transition is based on the behavior of the electron spins. As we can observe in Fig. 3, although the electrons dynamics is almost stochastic (due to multiple scattering and interference effects), their spins organize near the skyrmion core. This can be verified by measuring the topological $b$-field, defined in a similar way as the density $q$ of topological charge, but substituting $\boldsymbol{s}$ to $\boldsymbol{S}$ :

$$
b=\boldsymbol{n} \cdot \partial_{x} \boldsymbol{n} \times \partial_{y} \boldsymbol{n}, \quad \boldsymbol{n}=\boldsymbol{s} /|\boldsymbol{s}| ;
$$

represented in figure 3 by the color density. We verify that the transition is associated with the nucleation of a well localized electron vortex having a topological charge density opposite to the one of the background skyrmion (the white spots that appear near the skyrmion core at times $t=1100,1748,5936$ for the three values of the dissipation, respectively). We remark, that in spite of the dissipation, the characteristic length scale of these structures is comparable with a few lattice steps.

\section{CONCLUSION}

We investigated the transition between a skyrmion state and a ferromagnetic state driven by a spin polarized electron current. We demonstrated that the torque exerted by the itinerant spins modifies the distribution of the magnetization around the skyrmion core, and tends initially to reduce its size. When the system is dominated by the exchange interaction, a self-similar collapse of the core in a finite time produces, changing the topology of the system. In addition, this topological change strongly depends on the dissipation mechanism. In absence of dissipation the collapse time explicitly depends on the lattice cutoff. At variance, in the case where dissipation is effective, the singularity tends to regularize, and the life time of the skyrmion state reduces with increasing dissipation. However, the microscopic mechanism of topological change is in the dissipative case, similar to the nondissipative one (even if the time and length scales may differ). It is related to the appearance of a peculiar electronic structure possessing a net charge opposite to the one in the skyrmion. The synchronization of the fixed spins with this electron vortical structure, leads to the annihilation of the topological charge, which pass from its initial value $Q=-1$ (skyrmion state) to zero (homogeneous ferromagnetic state).

\section{ACKNOWLEDGEMENTS}

We thank R. G. Elías for useful discussions.

\section{REFERENCES}

V. G. Baryakhtar, B. A. Ivanov, A. L. Sukstanskii, and E. Yu. Melikhov. Soliton relaxation in magnets. Physical Review B, 56(2), 1997. URL http://link.aps.org/ abstract/PRB/v56/p619.

A. A. Belavin and A. M. Polyakov. Metastable states of two-dimensional isotropic ferromagnets. JETP Lett., 22 : 245-247, 1975. URL http: //www. jetpletters.ac.ru/ ps/1529/article_23383.shtml.

R. G. Elías and A. D. Verga. Topological changes of two-dimensional magnetic textures. Phys. Rev. B, 89 (13):134405, 2014. URL http://link.aps.org/doi/ 10.1103/PhysRevB.89.134405.

L. D. Landau and E. M. Lifshitz. On the theory of the dispersion of magnetic permeability in ferromagnetic bodies. Phys. Zeitsch. der Sow., 8:153-169, 1935. URL http://www.ujp.bitp.kiev.ua/index. php?item=j\&id=110. reprint: Ukr. J. Phys. 2008, Vol. 53, Special Issue, p.14-22.

S. Muhlbauer, B. Binz, F. Jonietz, C. Pfleiderer, A. Rosch, A. Neubauer, R. Georgii, and P. Boni. Skyrmion Lattice in a Chiral Magnet. Science, 323(5916):915-919, 2009. doi: 10.1126/science. 1166767. URL http://www.sciencemag.org/cgi/ content/abstract/323/5916/915.

D. C. Ralph and M. D. Stiles. Spin transfer torques. Journal of Magnetism and Magnetic Materials, 320(7): 1190-1216, 2008. URL http://www.sciencedirect. com/science/article/B6TJJ-4RFSD1M-2/2/ f35a2bc5e9c53f19f6883d74c20dbb69.

N. Romming, C. Hanneken, M. Menzel, J. E. Bickel, B. Wolter, K. von Bergmann, A. Kubetzka, and R. Wiesendanger. Writing and deleting single magnetic skyrmions. Science, 341(6146):636-639, 08 2013. URL http://www.sciencemag.org/content/ $341 / 6146 / 636$.

B. Van Waeyenberge, A. Puzic, H. Stoll, K. W. Chou, T. Tyliszczak, R. Hertel, M. Fahnle, H. Bruckl, K. Rott, G. Reiss, I. Neudecker, D. Weiss, C. H. Back, and G. Schutz. Magnetic vortex core reversal by excitation with short bursts of an alternating field. Nature, 444 (7118):461-464, 11 2006. URL http://dx.doi .org/10. 1038/nature05240.

V. E. Zakharov. Collapse of langmuir waves. Sov. Phys. JETP, 35(5):908-914, 1972. URL http://www.jetp. ac.ru/cgi-bin/e/index/e/35/5/p908?a=list.

S. Zhang and Z. Li. Roles of nonequilibrium conduction electrons on the magnetization dynamics of ferromagnets. Phys. Rev. Lett., 93:127204, 2004. URL http:// link.aps.org/abstract/PRL/v93/e127204. 